\title{
同轴线在电磁场理论中的应用
}

\author{
杨淑敏 \\ 喀什大学 物理与电气工程学院 \\ DOI:10.32629/er.v3i7.3011
}

\begin{abstract}
[摘 要] 同轴线问题主要是研究内外导体构成的双导体传输线问题, 是电磁场与电磁波的课程重要的 教学内容,其理论贯穿了整个电磁场理论的始终,在整个教学过程中发挥着重要作用, 本文通过对同轴线 的基本特性(如电容、电阻、阻抗、电感等)、能量、传输功率、同轴波导进行了分析讨论, 得出结论, 有 利于教师教学效果的提升和学生学习效率的提高。
\end{abstract}

[关键词] 同轴线; 电磁能量; 传输功率; 同轴波导; 解题思路

电磁场理论课是电子及电气信息专 业本科生必修专业基础课, 合格的电子、 电气信息类专业本科生应具备知识结构 的重要组成部分, 交叉学科生长点和新 兴边缘学科发展的基础 ${ }^{[1,2]}$ 。当今, 不论 是通信、雷达、广播、电视, 还是导航、 遥控遥测, 都是通过电磁波传递信息来 进行工作 ${ }^{[3]}$ 。因此以宏观电磁理论为基 础, 电磁信息的传输和转换为核心的电 磁场于电磁波工程技术将充分发挥其重 要作用 ${ }^{[4,5]}$ 。然而, 由于其理论体系严谨、 抽象复杂、要求一定的数学功底及推理 能力, 学生们学起来, 枯燥无味, 学习效 果不明显。在教学方面, 多数学者只是就 涉及同轴线问题的某一方面或模拟计算 特殊形状同轴线的电磁场进行研究 ${ }^{[6-11]}$, 研究内容不够具体, 总结不够全面。同轴 线问题贯穿整个电磁场理论的始终, 在 整个教学过程中发挥着重要作用, 如果 可以把这类问题搞懂, 学生们基本掌握 了整个体系, 可以使得学生更深入的理 解电磁场理论的内容, 脉络清唽, 为后续 课程垫定基础。为此, 我们将该类问题进 行总结归类、深入分析研究, 便于教师的 课堂教学和学生的自主学习。

\section{1 同轴线的基本特性}

1. 1 同轴线定义

同轴传输线是射频微波系统中最为 常用的传输线之一, 它不仅用于各种微 波设备间的连接线, 而且还常制作成各 种微波器件, 如同轴滤波器、同轴分配
器、同轴天线、同轴开关等。

同轴线是由内外两个不同半径的同 轴导体构成的, 一般中间充以电解质, 如 图1所示, 同轴线的内导体半径为a, 外导 体的内半径为 b, 外导体的外半径为 $c$, 内 外导体间充满介质 (参数为 $\mu, \varepsilon, \sigma)^{[12]}$ 。

下面我们分类讨论同轴线的问题, 按照电磁场理论知识的难易程度的递进 进行分析研究。首先探讨同轴线的电容 问题。

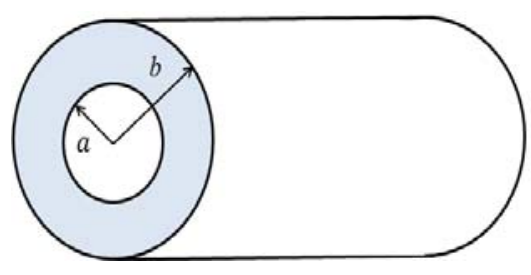

图1 同轴线示意图

\section{2 同轴线的电容}

生活中电容及其电容器, 尤其是平 行板电容器的应用极其广泛, 在两个相 距很近的平行板中间夹上一层绝缘物质 一电解质, 就组成一个简单的平行板电 容器。如果这个导体板是柱形的, 半径不 同, 内外导体间加有一层绝缘物质, 此即 同轴电容器。

设同轴线的内、外导体单位长度带 电量分别为 $+\lambda_{l}$ 和 $+\lambda_{l}$, 填充介质的 介电常数为 $\varepsilon$, 应用高斯定理可得到内 外导体间任一点的电场强度为

$$
\overrightarrow{E_{r}}=\overrightarrow{e_{r}} \frac{\lambda_{l}}{2 \pi \varepsilon r}
$$

内外导体间的电压为

$$
U=\int_{a}^{b} \vec{E}_{r} \cdot \vec{e}_{r} d r=\int_{a}^{b} \frac{\lambda_{l} 1}{2 \pi \varepsilon r}-d r=\frac{\lambda_{l}}{2 \pi \varepsilon} \ln -
$$

同轴线单位长度的电容为

$$
C_{l}=\frac{\lambda_{l}}{U}=\frac{2 \pi \varepsilon}{\ln (b / a)}
$$

由此我们可以得出结论, 单位长度 的电容仅和介质即电容器的尺寸相关, 而与通过的电流及电压无关, 即电容只 和其自身相关, 与此类似的还有绝缘电 阻。

\section{3 同轴线的绝缘电阻}

绝缘电阻是指用绝缘材料隔开的两 部分导体之间的电阻值, 其值大小反映 了两部分导体之间的绝缘强度, 对于电 线电缆而言, 绝缘电阻是十分重要的电 性能指标, 是评价电气安全的主要指标 之一, 如绝缘电阻失效是引起电动汽车 电安全事故、人员触电的主要原因 ${ }^{[13]}$ 。

内外导体间填充一种非理想介质, 设其介电常数为 $\varepsilon$, 电导率为 $\sigma$, 同轴线 内外导体间加恒定电压 $\mathrm{U} 0$, 介质中的漏 电流沿径向从内导体流到外导体 ${ }^{[14]}$ 。另 外, 内外导体中有轴向电流, 导体中存在 很小的轴向电场, 因而漏电介质中也存 在切向电场, 但 $E_{z} \ll E_{\gamma}$, 故可忽略。介质 中任一点处的漏电流密度为

$$
\vec{E}=\frac{\vec{J}}{\sigma}=\vec{e}_{r} \frac{\vec{J}}{2 \pi \sigma r}
$$


而内外导体间的电压为

$U_{0}=\int_{a}^{b} \vec{E} \cdot d \vec{r}=\int_{a}^{b} \frac{I}{2 \pi \sigma r} \cdot d r=\frac{I}{2 \pi \sigma} \ln \frac{b}{a}$

则同轴线单位长度的绝缘电阻 (漏 电阻) 为

$$
R_{1}=\frac{U_{0}}{I}=\frac{1}{2 \pi \sigma} \ln \frac{b}{a}
$$

由此, 我们计算得到了同轴线单位 长度的电阻, 其仅与电导率及电阻尺寸 有关, 与通过其电流及电压无关, 即电阻 也仅仅和其自身特征相关, 与此类似还 有电感。

\section{4 同轴线的电感}

电感的基本特征是“通直流阻交流”, 电容的基本特征就是 “通交流阻直流”, 在基本的直流电路中, 其用法和分析比 较简单, 但在交流电中, 电感和电容的阻 碍作用会随着交流电的特征而改变, 其 阻碍作用以阻抗来表示。电感线圈作为 电路中的基本元件, 在汽车中应用得非 常广泛, 例如电磁阀、电动机、发电机、 点火线圈等, 正确理解电感线圈的特性, 就为掌握这些部件的工作原理打下了良 好的基础。

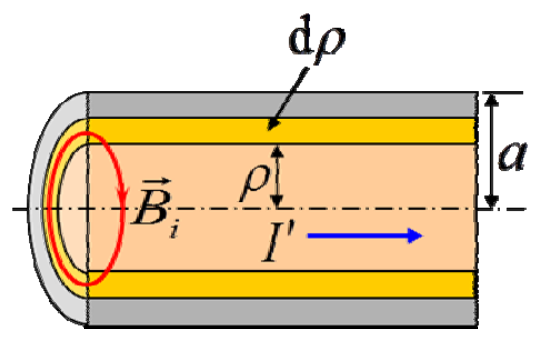

图2 同轴线剖面图

如图2所示, 设外导体的厚度可忽略 不计, 内、外导体之间是空气, 或聚乙烯 等电介质, 磁导率为 $\mu_{0}$, 内、外导体材料 一般是金属铜, 磁导率也是 $\mu_{0}$ 。

同轴线中的电流为I时, 根据安培环 路定理求得内导体中任意一点的磁感应 强度为

$$
\vec{B}_{i}=\vec{e}_{\phi} \frac{\mu_{0}}{2 \pi \rho} \frac{\pi r^{2}}{\pi a^{2}} I=\vec{e}_{\phi} \frac{\mu_{0} I r}{2 \pi a^{2}},(0<r<a)
$$

穿过由轴向为单位长度、宽为 $\mathrm{d} r$ 构 成的矩形面积元 $d \vec{S}=\vec{e}_{\phi} 1 \cdot d r=\vec{e}_{\phi} d r$ 磁 通为 $d \phi_{i}=\vec{B}_{i} \cdot d \vec{S}=\frac{\mu_{0} I r}{2 \pi a^{2}} d r$ 因为 $d \phi_{i}$ 与
这一部分磁通相交链的电流不是导体中 的全部电流 $I$, 而只是 $I$ 的一部分 $I^{\prime}$, 两者的关系为 ${ }^{[15]}$

$$
I^{\prime}=\frac{\pi r^{2}}{\pi a^{2}} I=\left(\frac{r}{a}\right)^{2} I
$$

所以, 与 $d \phi_{i}$ 相应的磁链为

$$
d \Psi_{i}=\frac{I^{\prime}}{I} d \phi_{i}=\frac{\mu_{0} I r^{3}}{2 \pi a^{4}} d r
$$

内导体中单位长度的自感磁链总量 为

$$
\Psi_{i}=\int d \Psi_{i}=\int_{0}^{a} \frac{\mu_{0} I r^{3}}{2 \pi a^{4}} d r=\frac{\mu_{0} I}{8 \pi}
$$

由此得到单位长度的内自感

$$
L_{i}=\frac{\Psi_{i}}{I}=\frac{\mu_{0}}{8 \pi}
$$

在内、外导体之间, 由安培环路定理 可得到任意一点磁感应强度为

$$
\vec{B}_{0}=\vec{e}_{\phi} \frac{\mu_{0} I}{2 \pi r}, \quad(a<r<b)
$$

故

$$
d \Psi_{0}=\frac{\mu_{0} I}{2 \pi r} d r
$$

$\Psi_{0}=\int d \Psi_{0}=\int_{a}^{b} \frac{\mu_{0} I}{2 \pi r} d r=\frac{\mu_{0} I}{2 \pi} \ln \frac{b}{a}$

由此得到单位长度的外自感

$$
L_{0}=\frac{\Psi_{0}}{I}=\frac{\mu_{0}}{2 \pi} \ln \frac{b}{a}
$$

同轴线单位长度的自感为

$$
L=L_{i}+L_{0}=\frac{\mu_{0}}{8 \pi}+\frac{\mu_{0}}{2 \pi} \ln \frac{b}{a}
$$

由此我们得出同轴线电感的计算式, 其也仅仅与磁导率和同轴线的尺寸有关, 而与通过的磁场及电流是无关的, 即电 感仅仅与自身特征有关, 这个特点和我 们前面分析的电容和电阻相似。此外, 我们进一步讨论了同轴线的阻抗问题。

1.5 同轴线的特性阻抗

传输线的特性阻抗是其特性参数之 一, 定义为传输线上行波电压与行波电 流之比。

$$
z_{0}=\frac{U^{+}}{I^{+}}=-\frac{U^{-}}{I^{-}}=\sqrt{\frac{R_{1}+j \omega L_{1}}{G_{1}+j \omega C_{1}}}
$$

对于无损耗传输线, $R 1=0 、 G 1=0$, 则

$$
z_{0}=\sqrt{\frac{L_{1}}{C_{1}}}
$$

而对于同轴线, 单位长度的电容

$$
C_{1}=\frac{2 \pi \varepsilon}{\ln (b / a)}
$$

单位长度的电感

$$
L_{1}=\frac{\mu}{2 \pi} \ln \frac{b}{a}
$$

所以同轴线的特性阻抗为

$$
z_{0}=\sqrt{\frac{L_{1}}{C_{1}}}=\frac{60}{\sqrt{\varepsilon}} \ln \frac{b}{a}
$$

上式即我们求得的同轴线的特性阻 抗, 由上式可以得出特性阻抗也仅仅与 电介质及自身尺寸有关, 而与行波电压 和行波电流无关, 即特性阻抗仅与其自 身特征有关, 并且以上我们所讨论的电 容、电阻及电感都具有此特征。经过总 结归纳类似特性、举一反三, 可以使得学 生对类似问题达到触类旁通的目的, 印 象深刻, 学以致用, 使本身枯燥繁杂的问 题变得清晰、简单明了。除了以上同轴 线问题的研究, 我们还讨论了同轴线能 量的问题。

\section{2 同轴线内能量的储存}

2.1 同轴线内储存的电场能量

首先讨论同轴线电场能量的问题。 设同轴线的内、外导体单位长度带电量 分别为和, 填充介质的介电常数为, 应用 高斯定理可得到内外导体间任一点的电 场强度为

$$
\vec{E}_{r}=\vec{e}_{r} \frac{\lambda_{l}}{2 \pi \varepsilon r}
$$

内外导体间的电压为

$$
U=\int_{a}^{b} \vec{E}_{r} \cdot \vec{e}_{r} d r=\frac{\lambda_{l}}{2 \pi \varepsilon} \int_{a}^{b} \frac{1}{r} d r=\frac{\lambda_{l}}{2 \pi \varepsilon} \ln \frac{b}{a}
$$

则同轴线单位长度的电容为

$$
C=\frac{\lambda_{l}}{U}=\frac{2 \pi \varepsilon}{\ln (b / a)}
$$

则得同轴线单位长度的静电储能为

$$
W_{e}=\frac{1}{2} \int_{V} \varepsilon E^{2} d V=\frac{1}{2} \int_{a}^{b} \varepsilon\left(\frac{\lambda_{l}}{2 \pi \varepsilon r}\right)^{2} 2 \pi r d r=\frac{\lambda_{l}^{2}}{4 \pi \varepsilon} \ln \left(\frac{b}{-a}\right)
$$


此即同轴线单位长度的静电场能量, 由上式我们发现其除了与电介质及其尺 寸有关, 还与导体内储存的电荷有关, 这 一点和我们前面讨论的电容、电感及特 性阻抗问题不同, 即其不但与自身特征 有关还与储存的电荷有关。具有类似特 征的还有同轴线的磁场能量问题。

2.2 同轴线内储存的磁场能量

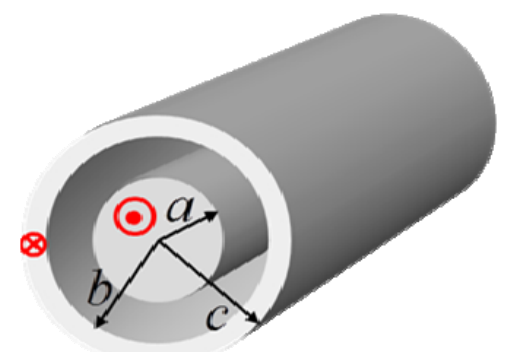

图3 同轴线内部电流分布示意

设内外导体之间填充的介质以及导 体的磁导率均为 $\mu 0$, 电流为 $I$, 其电流分 布如图3所示。根据安培环路定理可得磁 场分布

$$
\begin{gathered}
\vec{H}_{1}=\vec{e}_{\phi} \frac{I r}{2 \pi a^{2}}, \quad(0 \leq r \leq a) \\
\vec{H}_{2}=\vec{e}_{\phi} \frac{I}{2 \pi r}, \quad(a \leq r \leq b) \\
\vec{H}_{2}=\vec{e}_{\phi} \frac{I}{2 \pi r} \frac{c^{2}-r^{2}}{c^{2}-b^{2}}, \quad(b \leq r \leq c)
\end{gathered}
$$

由此即可求出三个区域单位长度内 的磁场能量分别为

$$
\begin{aligned}
\mathrm{W}_{m 1} & =\frac{\mu_{0}}{2} \int_{0}^{a} \vec{H}_{1} 2 \pi r d r=\frac{\mu_{0}}{2} \int_{0}^{a}\left(\frac{I r}{2 \pi a^{2}}\right)^{2} 2 \pi r d r=\frac{\mu_{0} I^{2}}{16 \pi} \\
\mathrm{W}_{m 2} & =\frac{\mu_{0}}{2} \int_{a}^{b} \vec{H}_{2} 2 \pi r d r=\frac{\mu_{0}}{2} \int_{a}^{b}\left(\frac{I}{2 \pi r}\right)^{2} 2 \pi r d r=\frac{\mu_{0} I^{2}}{4 \pi} \ln \frac{b}{a} \\
\mathrm{~W}_{m 3} & =\frac{\mu_{0}}{2} \int_{b}^{c} \vec{H}_{3} 2 \pi r d r=\frac{\mu_{0}}{2} \int_{b}^{c}\left(\frac{I}{2 \pi r c^{2}-b^{2}}\right)^{2} 2 \pi r d r \\
& =\frac{\mu_{0} I^{2}}{4 \pi}\left[\frac{c^{4}}{\left(c^{2}-b^{2}\right)^{2}} \ln \frac{c}{b}-\frac{3 c^{2}-b^{2}}{4\left(c^{2}-b^{2}\right)}\right]
\end{aligned}
$$

同轴线单位长度储存的总磁场能量 为

$$
\mathrm{W}_{m}=\mathrm{W}_{m 1}+\mathrm{W}_{m 2}+\mathrm{W}_{m 3}
$$

$=\frac{\mu_{0} I^{2}}{16 \pi}+\frac{\mu_{0} I^{2}}{4 \pi} \ln \frac{b}{a}+\frac{\mu_{0} I^{2}}{4 \pi}\left[\frac{c^{4}}{\left(c^{2}-b^{2}\right)^{2}} \ln \frac{c}{b}-\frac{3 c^{2}-b^{2}}{4\left(c^{2}-b^{2}\right)}\right]$
此即我们讨论的同轴线的磁场能量, 很显然其不但与磁导率及其同轴线尺寸 有关, 还与通过的电流有关, 即磁场能量 除了和其自身特征有关还与通过的电流 有关。由此, 我们进一步讨论了同轴线的 功率问题。

\section{3 同轴线中传输的功率}

设两导体间的电压为U, 其间填充均 匀的理想介质, 导体中流过的电流为 $\mathrm{I}$ 。 理想导体的情况下, 同轴线中的传输功 率以距对称轴为 $r$ 的半径作一圆周, 应用 安培环路定理, 由对称性得

$$
2 \pi r H_{\theta}=I
$$

因而

$$
H_{\theta}=\frac{I}{2 \pi r}
$$

设内导体单位长度得电荷为, 应用 高斯定理, 由对称性可得

$$
2 \pi r E_{r}=\frac{\lambda_{l}}{\varepsilon}
$$

因而

$$
E_{r}=\frac{\lambda_{l}}{2 \pi \varepsilon r}
$$

能流密度为

$\vec{S}=\vec{E} \times \vec{H}=E_{r} H_{\theta} \vec{e}_{z}=\frac{I \lambda_{l}}{4 \pi^{2} \varepsilon r^{2}} \vec{e}_{z}$

$\vec{e}_{z}$ 为沿导线轴向单位矢量。

由于导线间得电压

$$
U=\int_{a}^{b} E_{r} d r=\frac{\lambda_{l}}{2 \pi \varepsilon} \ln \frac{b}{a}
$$

因而

$$
\vec{S}=\frac{U I}{2 \pi \ln (b / a)} \frac{1}{r^{2}}
$$

电磁能量在两导体之间的介质中沿 轴方向流动, 即由电源向负载。

$\mathrm{P}=\int_{S} \vec{S} \cdot \vec{e}_{z} d S=\int_{a}^{b} \frac{U I}{2 \pi r^{2} \ln (b / a)} 2 \pi r d r=U$

UI为通常电路问题中得传输功率表 示式。可见, 同轴线传输的功率是通过内 外导体间的电磁场传递到负载, 而不是 经过导体内部传递的。

当导体的电导率 $\sigma$ 为有限值时, 导 体内部存在沿电流方向的电场

$$
\vec{E}=\frac{\vec{J}}{\sigma}=\frac{I}{\pi a^{2} \sigma} \vec{e}_{z}
$$

根据边界条件, 在内导体表面上电 场的切向分量连续。因此, 在内导体表面 外侧的电场除有径向分量还有切向分 量,

$$
\left.\vec{E}\right|_{r=a}=\frac{U}{\operatorname{aln}(b / a)} \vec{e}_{r}+\frac{I}{\pi a^{2} \sigma} \vec{e}_{z}
$$

磁场则仍为

$$
\left.\vec{H}\right|_{r=a}=\vec{e}_{\phi} \frac{I}{2 \pi a}
$$

内导体表面外侧的坡印廷矢量为 $\left.\vec{S}\right|_{r=a}=\left.(\vec{E} \times \vec{H})\right|_{r=a}=-\frac{I}{2 \pi^{2} a^{3} \sigma} \vec{e}_{r}+\frac{U I}{2 \pi a^{2} \ln (b / a)} \vec{e}_{z}$

由此可见, 内导体表面外侧的坡印 廷矢量既有轴向分量, 也有径向分量。

进入每单位长度内导体的功率为 $\mathrm{P}=\left.\int_{S} \vec{S}\right|_{r=a} \cdot\left(-\vec{e}_{r}\right) d S=\int_{0}^{1} \frac{I^{2}}{2 \pi^{2} a^{3} \sigma} 2 \pi a d z=\frac{I^{2}}{\pi a^{2} \sigma}=R I^{2}$

式中 $\mathrm{R}=I^{2} /\left(\pi a^{2} \sigma\right)$ 是单位长度内导 体的电阻。由此可见, 进入内导体中的功 率等于这段导体的的焦耳损耗功率。

以上分析表明, 电磁能量是通过电 磁场传输的, 导体仅起着定向引导电磁 能流的作用。当导体的电导率为有限制 时, 进入导体中的功率全部被导体所吸 收, 成为导体中的焦耳热损耗功率。此即 同轴线的功率问题。为了进一步更深入 的讨论同轴线的问题, 使学生能够更深 刻的理解并掌握此类问题, 我们又讨论 了同轴波导中的电磁场问题。

\section{4 同轴波导中的电磁场}

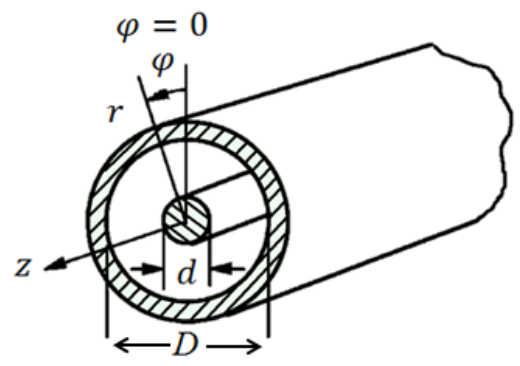

图4 同轴波导示意图

同轴波导是一种由内外导体构成的 双导体导波系统, 也称为同轴线, 其形状 如图4所示。由于同轴线是双导体波导, 因 此它既可以传播TEM波, 也可以传播TE波、 TM波。设电磁波沿+Z方向传播, 相应的场 为时谐场, 波导内电磁场的复数形式为 ${ }^{[14]}$ 


$$
\vec{E}(\rho, \phi, z)=\vec{E}(\rho, \phi) e^{-\gamma z}
$$

$$
\vec{H}(\rho, \phi, z)=\vec{H}(\rho, \phi) e^{-\gamma z}
$$

对于TEM波, $E_{z}=0, H_{z}=0$ ， 而磁力线是闭合曲线, 电场和磁场都在 横截面内, 即 $\vec{H}=\vec{e}_{\phi} H_{\phi}, \vec{E}=\vec{e}_{\rho} E_{\rho}$, 故将麦克斯韦方程

$$
\begin{aligned}
\nabla \times \vec{H} & =j \omega \varepsilon \vec{E} \\
\nabla \times \vec{E} & =j \omega \mu \vec{H}
\end{aligned}
$$

在圆柱坐标系中展开, 得

$$
\gamma H_{\phi}=j \omega \varepsilon E_{\rho}
$$

$$
\frac{1}{\rho} \frac{\partial}{\partial \rho}\left(\rho H_{\phi}\right)=0
$$

$\gamma E_{\rho}=j \omega \mu H_{\phi}$

$$
\frac{1}{\rho} \frac{\partial}{\partial \rho}\left(\rho E_{\rho}\right)=0
$$

考虑沿 $+Z$ 方向的传播因子 $e^{-\gamma z},(2)$ 式的解为

$$
H_{\phi}=\frac{H_{m}}{\rho} e^{-\gamma z}
$$

将上式代入 (1式) 得

$$
E_{\rho}=\frac{\gamma}{j \omega \varepsilon} H_{\phi}=\frac{\gamma}{j \omega \varepsilon} \frac{H_{m}}{\rho} e^{-\gamma z}
$$

同轴波导中TEM模的场分布如图5所示
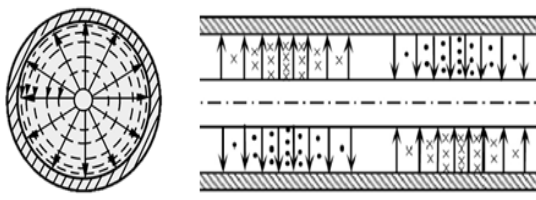

图5 同轴线TEM模的示意图 由于TEM波有式

$$
\gamma_{T E M}^{2}+k^{2}=0
$$

从而得TEM波得传播常数

$$
\gamma=\gamma_{\text {TEM }}=j k=j \omega \sqrt{\mu \varepsilon}=j \beta
$$

式中 $\beta=\omega \sqrt{\mu \varepsilon}$ 为 TEM波的相位常

数。其余传播特性参数分别为:

相速度

$$
v_{p}=\frac{\omega}{\beta}=\frac{1}{\sqrt{\varepsilon \mu}}
$$

波阻抗

$$
Z_{T E M}=\frac{E_{r}}{H_{\phi}}=\frac{\gamma}{j \omega \varepsilon}=\sqrt{\frac{\mu}{\varepsilon}}=\eta
$$

从以上的分析可知, TEM波是无色散 波, 其截至波数

$$
k_{c}=\sqrt{\gamma^{2}+k^{2}}=0
$$

即 $\lambda_{c}=\infty$ 。因此, 同轴波导中的主 模是TEM模。

同轴波导中传输TEM模时, 其传输功 率为

$\mathrm{P}=\frac{1}{2} R_{e} \int_{S}\left(\vec{E} \times \vec{H}^{*}\right) \cdot d \vec{S}=\frac{1}{2} R_{e} \int_{a}^{b}\left(\vec{E}_{t} \times \vec{H}_{t}^{*}\right) \cdot \vec{e}_{z} d S$

$=\frac{1}{2} \int_{a}^{b}\left(E_{r} \cdot H_{\phi}^{*}\right) 2 \pi r d r=\pi \frac{\gamma}{j \omega \varepsilon}\left|H_{m}\right|^{2} \ln \frac{b}{a}$

$$
=\pi \sqrt{\frac{\mu}{\varepsilon}}\left|H_{m}\right|^{2} \ln \frac{b}{a}=\frac{\pi}{\eta}\left|E_{m}\right|^{2} \ln \frac{b}{a}
$$

同轴波导中传播TEM模时, 在 $\mathrm{r}=\mathrm{a}$ 处 电场最大, 且等于

$$
\left|E_{a}\right|=\sqrt{\frac{\mu}{\varepsilon}} \frac{H_{m}}{a}=\frac{\eta H_{m}}{a}=\frac{E_{m}}{a}
$$

若假设该处的电场强度 $\left|E_{a}\right|$ 等于同 轴波导中所填充媒质的击穿电场强度 $E_{b r}$, 则击穿时有 $\left|E_{m}\right|=E_{b r} a$, 将其代入 式 (5), 得同轴波导传输TEM模时的功率 容量为

$$
\mathrm{P}_{b r}=\frac{\pi a^{2} E_{b r}^{2}}{\eta} \ln \frac{b}{a}
$$

在实际应用中, 同轴波导都是以TEM 模 (主模) 方式工作 ${ }^{[16]}$ 。但是, 当工作频 率过高时, 在同轴波导中还将出现一系 列的高次模: TM模和TE模。同轴波导中 的 TM模和TE模的分析方法相似, 即在给 定的边界条件下求解 $E_{z}$ 或 $H_{z}$ 满足的 波动方程, 从而可以得到同轴波导不同 $T M_{m n}$ 模和 $T E_{m m}$ 模的场分布以及相应模 式的截止波长 $\left(\lambda_{c}\right)_{m n}$ 。

根据计算得到同轴波导中 $T E_{11}$ 和 $T M_{01}$ 的截止波长分别为 ${ }^{[16]}$

$$
\left(\lambda_{c}\right)_{T E_{11}} \approx \pi(b+a)
$$

$$
\left(\lambda_{c}\right)_{T M_{01}} \approx 2(b-a)
$$

于是, 同轴波导几个较低阶的模式 分布如图6所示

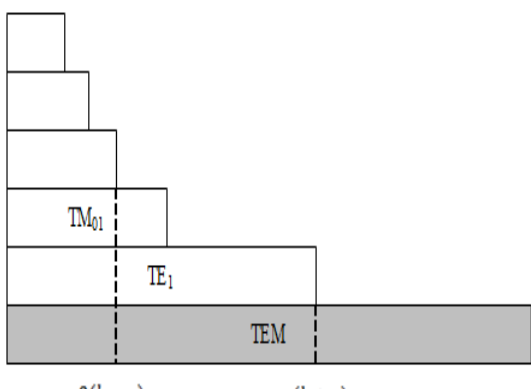

$2(b-a) \quad \pi(b+a)$

图6 同轴线中的高次模分布 ${ }^{[16]}$

$$
\lambda_{\min } \geq\left(\lambda_{c}\right)_{T E_{11}} \approx \pi(b+a)
$$

$\mathrm{a}+\mathrm{b} \leq \frac{\lambda_{\min }}{\pi}$

式 (6) 给出了 $a+b$ 的取值范围, 要 最终确定尺寸, 还必须确定的 $a / b$ 值 [16]。可以根据实际需要选择该值的大 小。例如, 当要求功率容量最大时选择 $a / b=1.65$, 当要求传输损耗最小时选择 $a / b=3.59$, 当要求耐压最高时选择 $a / b=2.72$ 。以上即为我们研究的同轴线 的波导问题, 至此电磁场理论中关于同 轴线的问题已详细充分讨论。通过此类 问题的研究和讨论, 对于电磁场问题知 识得到升华, 难以理解的问题豁然开朗。

\section{5 结语}

电磁场理论是低频电路理论的进一 步发展, 低频电路理论是它的特例。同轴 线问题贯穿整个电磁场理论的始终, 在 整个教学过程中发挥着重要作用, 文章 对同轴线问题中涉及的电容、电阻、阻 抗、电感等基本特性、能量、传输功率、 同轴波导等进行了总结, 通过这些特性 的分析及对比研究, 可以促进学生加深 对知识的理解, 有利于提高学生学习效 率; 从教学的角度来看, 教师在教学过程 中通过各散乱知识点的总结, 可以调动 学生的联想记忆, 使前后知识连贯对应, 有利于教学效果的提升。

\section{[参考文献]}

[1]赵家升, 杨显清, 王园.《电磁场与 电磁波》(第 3 版) 教学指导书 $[M]$. 高等教 育出版社,2003. 


\title{
探析幼儿园区角活动的目的和意义及如何开展
}

\author{
刘倩 \\ 射洪市第三幼儿园 \\ DOI:10.32629/er.v3i7.2985
}

\begin{abstract}
[摘 要] 区角活动是幼儿教育中独特的教育方式,这种活动既能把幼儿教育中课内和课外的行为、情 感、技能有机联系起来, 还能将教育者的教育意图渗透其中, 并且还具有自主性、自选性、小组性, 是落 实儿童个性发展的最有效措施。那么在幼儿园如何才能更好地开展区域活动,让幼儿园区角活动取得更 大成效,下面是笔者结合实际工作,就此问题浅谈几点看法。
\end{abstract}

[关键词]幼儿园; 区角活动; 有效开展

\section{引言}

幼儿园区角活动是幼儿在幼儿园内 进行的重要的游戏活动之一, 区角活动 从字面上理解就是在某个角落进行的活 动, 实际上是一种自主活动方式。指教师 根据一定的活动空间和活动材料, 幼儿 在丰富的环境中进行自主、自由的探索 性活动和个性化学习。追溯区域活动的 发展历史, 20 世纪 90 年代初, 海外留学人 员把区域活动模式介绍到中国。如今, 区域活动已经在幼儿园得到了很大程度 的重视。大部分幼儿园都纷纷开展区角 活动, 但是在实际开展的区角活动过程 中仍然存在各方面的问题。

\section{1 开展幼儿园区角活动的重要}

[2]周希朗.电磁场 $[M]$. 电子工业出 版社,2008.

[3]郭瑜, 虞致国.电磁场与电磁波仿 真实验教学研究 [J]. 无锡职业技术学院 学报,2018,17(02):33-36.

[4]贾晋辉. 电磁场与电磁波在电子 通信技术中的应用[ J ] 军民两用技术与 产品,2016,(14):71.

[5]胡章迪.电磁场与电磁波在电子 通信技术中的应用 [J]. 科技 风,2015,12(20):116.

[6]王福谦.方同轴线中的 TEM 波及 其特性阻抗 [J]. 长治学院学 报,2013,(02):20-22.

[7]姚虹.非理想导体同轴线中的电

\section{目的与意义}

1.1 1 区角活动促进幼儿的个性发展

幼儿园区角活动能够适应每个幼儿 不同个性的发展, 由于每个幼儿的个性 特点, 能力倾向, 原有经验等多方面都有 所不同。区角活动自由自选自发性等特 点为幼儿个性发展提供可能。区角活动 与传统的教学模式不同, 将区域大概分 为角色区, 建构区, 表演区, 益智去娃娃 家, 美工区等几个大类。

\section{2提高幼儿的主观选择能动性}

实施幼儿园区角活动的具体过程, 就是幼儿和老师之间展开的有效沟通。 对于教师而言, 只有时时刻刻将幼儿作 为活动的主体, 并对他们开展的活动进

磁场和能流密度 [J]. 内蒙古科技与经 济,2007,(22):92-93.

[8]王福谦. 内方外圆同轴传输线中 TEM 波的场结构及其特性阻抗[J]. 山西大 学学报:自然科学版,2013,36(3):410-415.

[9]李庆和. 射频同轴电缆特性阻抗 的辨析 [J]. 光纤与电缆及其应用技 术,2017,(1):1-4.

[10]周军霞.同轴电缆特性阻抗测 试方法及应用研究 [J]. 机电工程技 术,2010,39(6):35-38.

[11]骆新江, 张忠海.同轴特性阻抗 电磁场综合实验 [J]. 实验室研究与探 索,2019,38(11):5-10.
行合理指导, 才能培养幼儿的动手能力。 其中, 最具有现代化意义的就是, 极大地 提高了幼儿的主观选择能动性。

\section{2 幼儿园区角活动开展的策略}

2.1对幼儿园区角活动的环境展开 科学设置

幼儿园区角活动环境的设置, 不仅 仅是确保区角活动顺利实施的前提条件, 同样还是创设优良环境的基础。这就需 要合理科学规划活动区角的范围, 比如 舞蹈区、休息区以及活动区等。为最大 限度地提高幼儿园的空间使用率, 进而 可以为幼儿构建出一个可以自由自在活 动的场所, 必须要注意以下几点:

2.1.1需要考虑幼儿的需求

电磁波 [M].国防工业出版社,2003.

[13]项晟晧.绝缘电阻测试的分析 研究[J].北京汽车,2019,(1):3-7.

[14]张厚.电磁场与电磁波及其应 用 $[M]$. 西安电子科技大学出版社, 2012 .

[15]曹祥玉,高军,曾越胜,等.电磁场 与电磁波 $[M]$. 西安电子科技大学出版 社,2007.

[16]谢处方, 饶克谨.电磁场与电磁 波(第四版)[M].高等教育出版社,2006.

\section{作者简介：}

杨淑敏(1981--), 汉族, 女, 山东莘县 人, 喀什大学物理与电气工程学院, 副教 授,物理学教育。 